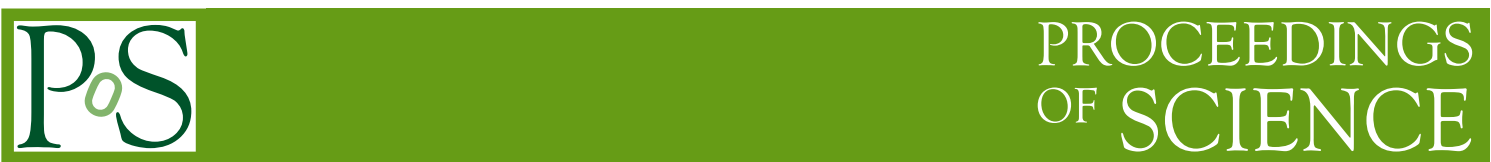

\title{
Multiboson measurements with Run 2 data at CMS
}

\author{
Alessandro Da Rold ${ }^{a, b, *}$, on behalf of the CMS collaboration \\ ${ }^{a}$ Università degli studi di Trieste, \\ Piazzale Europa 1, Trieste, Italy \\ ${ }^{b}$ INFN Sezione di Trieste, \\ via Valerio 2, Trieste, Italy \\ E-mail: alessandro.da.rold@cern.ch
}

\begin{abstract}
Processes with more than one boson in the final state are interesting because they can provide information on different aspects of Electro-Weak High Energy Physics. The non-abelian structure of the theory can be tested through the measurement of the strength of the couplings, while possible deviations from the theoretical prediction can hint to new physics effects. Some of the most recent results obtained by the CMS collaboration exploiting the data collected between 2016 and 2018 are presented in this note. The very high statistics that has been recorded, close to $140 \mathrm{fb}^{-1}$, opens the path to the precision study of processes that were not accessible until now.
\end{abstract}

The Eighth Annual Conference on Large Hadron Collider Physics-LHCP2020

25-30 May, 2020

online

\footnotetext{
${ }^{*}$ Speaker
} 


\section{Introduction and motivation}

The motivations for the study of multiboson processes are manifold. The non-Abelian gauge structure of the electroweak sector of the Standard Model (SM) allows the bosons to self-interact. The strength of these interactions is set by the values of triple (TGC) and quartic gauge couplings (QGC). From the precise measurement of the cross section of multiboson processes, it is possible to extract the values of these couplings and therefore test the consistency of the electroweak sector of the SM itself. Moreover, many multiboson final states are backgrounds for other precision analyses and for searches for new particles. A precise description of the contribution of these SM processes is therefore fundamental in order to maximise the sensitivity of future analyses.

Measuring the strength of the couplings is also a powerful indirect search for new particles. The potential presence of anomalous gauge couplings (aGC) can be interpreted in the framework of Effective Field Theories (EFT). The SM Lagrangian can be expanded by including terms with dimension higher than four

$$
\mathcal{L}_{\mathrm{aGC}}=\mathcal{L}_{\mathrm{SM}}+\sum_{i} \frac{f_{i}}{\Lambda^{d-4}} O_{i}+\ldots
$$

where $O_{i}$ are the operators that modify the lagrangian, $\Lambda$ is the energy scale of the new physics, $d$ is the dimension of the operators and $f_{i}$ are the Wilson coefficients that are related to the strength of the coupling. By measuring the differential cross sections of the multiboson processes, the Wilson coefficients normalised to the energy scale can be derived and if found to be different from zero they can hint to the presence of new particles.

The experimental measurement of multiboson processes is challenging in many ways. First of all, the cross sections are very small. Multiboson processes can be divided into two categories: quantum chromo-dynamics (QCD) induced ones and vector-boson scattering (VBS) ones. The former have typical cross sections of the order of hundreds of femtobarns, while the latter have typical cross sections at least one order of magnitude smaller. For this reason, to perform multiboson analyses, it is necessary to have a high statistics, such as the one collected by the Compact Muon Solenoid (CMS) experiment during the LHC Run 2, approximately equal to $140 \mathrm{fb}^{-1}$. The presence of many particles in the final state and the high level of precision that must be reached require a optimal particle reconstruction and identification.

\section{Measurement of the $\mathrm{W}^{+} \mathbf{W}^{-}$process}

The production of a $\mathrm{W}^{+} \mathrm{W}^{-}$pair has been studied by the CMS experiment by using the data collected in 2016 at a centre of momentum energy of $13 \mathrm{TeV}$, corresponding to an integrated luminosity of $36.9 \mathrm{fb}^{-1}$ [1]. The goal of the analysis was to measure the cross section of the process and set limits on anomalous gauge couplings.

A pair of $\mathrm{W}^{+} \mathrm{W}^{-}$bosons can be produced by s-channel or t-channel $\mathrm{q} \overline{\mathrm{q}}$ annihilation, by $\mathrm{gg} \rightarrow \mathrm{W}^{+} \mathrm{W}^{-}$at higher order in QCD and by Higgs decay (even though with a cross section ten times smaller than the other processes). The process has been studied only for the leptonic decay of both the $\mathrm{W}$ bosons in order to have a signal as clean as possible. Two different approaches have been followed for the event selection: a "standard" one, based on a cut analysis, and one based on random forest classifiers (RFC). For the sequential cut analysis, two opposite charge isolated leptons with 

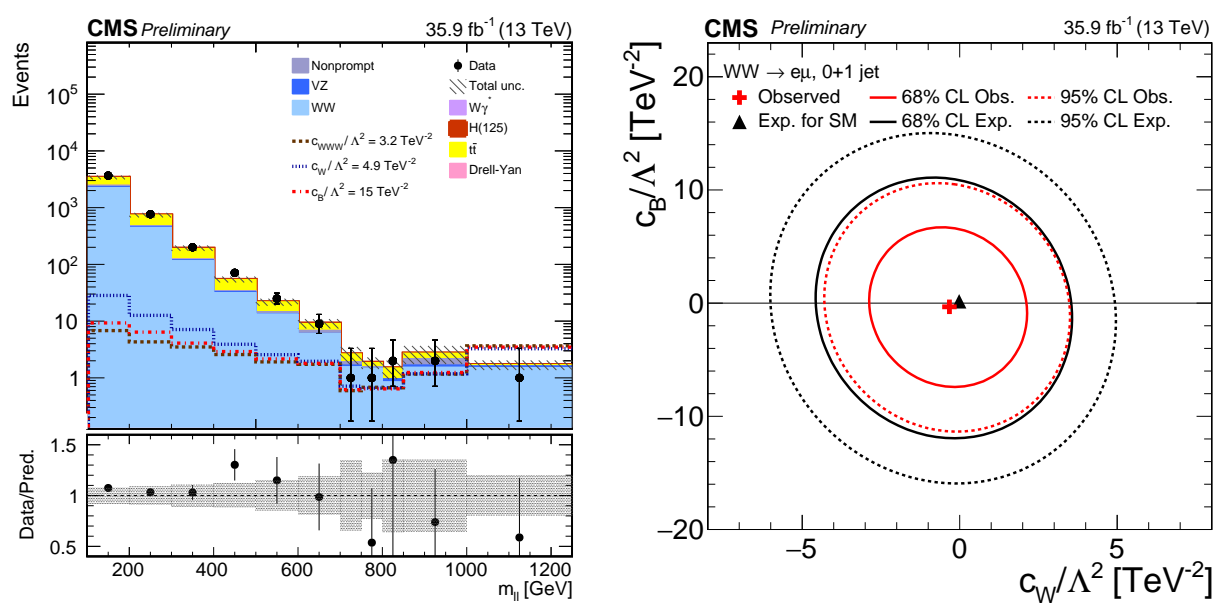

Figure 1: Invariant mass of the two leptons (left) for data (black dots) and simulations (filled area); the dotted lines are the theoretical expectations for different values of the aGC parameters. Observed and expected limits on two couplings (right).

an invariant mass of at least $20 \mathrm{GeV}$ were required alongside a missing transverse momentum of at least $20 \mathrm{GeV}$ to take into account the presence of the neutrinos. A multi-variate analysis (MVA) has been implemented to better reduce the background contamination. The contribution of the two main backgrounds, Drell-Yan and $t \bar{t}$ events, has been estimated in control regions.

A RFC is an aggregate of binary decision trees trained independently and in parallel. The greatest advantage of this approach is to mitigate data overfitting as much as possible. As for the sequential cut analysis, two opposite charge isolated leptons with invariant mass greater than $30 \mathrm{GeV}$ were required. In order to maximise the power to remove background contributions, two different RFC have been built: one to remove the contribution of Drell-Yan events and another one to remove $t \bar{t}$ contributions. The signal efficiency and purity of the RFC approach are higher than the ones resulting from the sequential cut analysis.

The total cross section has been measured with both methods and found to be in agreement with the predicted SM value. The main systematic uncertainties affecting the result come from the normalisation of Drell-Yan and $\bar{t} \bar{t}$ backgrounds. The jet multiplicity has been measured by exploiting the RFC analysis. The unfolded results, important to probe theoretical calculations and event generators accuracy, are in agreement with the prediction. Limits on dimension-6 operators with massive boson fields $\left(O_{W W W}, O_{W}, O_{B}\right)$ have been extracted from the invariant mass distribution of the two leptons. The results, also shown in Figure 1, are in agreement with the SM prediction.

\section{Measurement of the triboson production}

The three boson processes have been studied by the CMS collaboration with data collected between 2016 and 2018 at a centre of momentum energy of $13 \mathrm{TeV}$ corresponding to $137 \mathrm{fb}^{-1}$ [2]. Both the total and all the individual VVV cross sections have been measured in the $\mathrm{W}^{ \pm} \mathrm{W}^{ \pm} \mathrm{W}^{\mp} \rightarrow$ $1^{ \pm} 1^{ \pm} 2 v q q^{\prime}, \mathrm{W}^{ \pm} \mathrm{W}^{ \pm} \mathrm{W}^{\mp} \rightarrow 1^{ \pm} 1^{ \pm} 1^{\mp} 3 v, \mathrm{~W}^{ \pm} \mathrm{W}^{\mp} \mathrm{Z} \rightarrow 1^{ \pm} 1^{\mp} 2 v 1^{ \pm} 1^{\mp}, \mathrm{W}^{ \pm} \mathrm{ZZ} \rightarrow 1^{ \pm} v 2\left(1^{ \pm} 1^{\mp}\right)$ and $\mathrm{ZZZ} \rightarrow$ $3\left(1^{ \pm} 1^{\mp}\right)$ channels. 


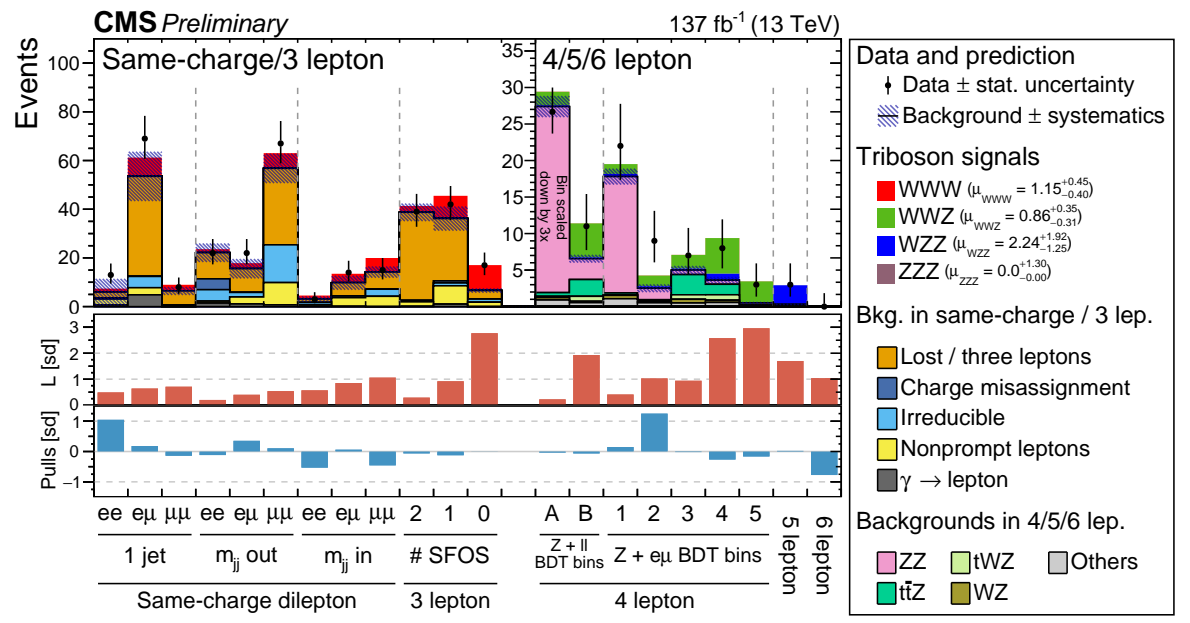

Figure 2: number of events in each VVV production and decay channel. The expected significance for each signal channel is presented in the middle plot, while on the bottom plot the difference between observed and predicted events is reported.

The WWW channel is the one with the greater number of events, but also the one with the higher background contribution. In order to have as clean a selection as possible, 2 same sign leptons and at least one jet have been required for the $1^{ \pm} 1^{ \pm} 2 v q^{\prime}$ channel, while 0,1 or 2 same flavour opposite charge lepton pairs with invariant mass incompatible with the $\mathrm{Z}$ invariant mass have been selected for the fully leptonic channel. The main backgrounds come from events with a lost lepton, same sign leptons coming from other processes (such as $\mathrm{t} \overline{\mathrm{t}}$ ) with one jet and nonprompt leptons. The background is much smaller for the channels with one or more $\mathrm{Z}$ bosons, where 1, 2 or 3 same flavour opposite charge lepton pairs compatible with the $\mathrm{Z}$ mass are selected. The cleaner signal, however, is obtained at the price of much smaller cross sections and branching ratios.

The observed and measured number of events for all the analysed channels is presented in Figure 2. The nonprompt lepton background has been estimated from data in control regions obtained by exploiting isolation variables. BDTs have been trained with simulated background and signal events in each channel in order to reduce the background contributions as much as possible.

The cross sections and signal strength have been measured for all the channels and found to be in agreement with the SM prediction. The BDT analysis allowed to have a significance of more than $3 \sigma$ for the WWW and the WWZ channels and of $5.7 \sigma$ for all the combined channels. This constitutes the first evidence for these two channels and the first observation of the combined heavy triboson production by the CMS collaboration.

\section{References}

[1] The CMS collaboration, Studies of $\mathrm{W}^{+} \mathrm{W}^{-}$production at $\sqrt{s}=13 \mathrm{TeV}$, CMS-PAS-SMP-18004 (2020)

[2] The CMS collaboration, Observation of heavy triboson production in leptonic final states in proton-proton collisions at $\sqrt{s}=13 \mathrm{TeV}$, CMS-PAS-SMP-19-014 (2020) 